\title{
¿Que hacer en los casos que dan positivo a metadona en los controles de drogas en carretera? La experiencia de España
}

\section{What to do when drivers test positive for methadone in roadside drug tests? The Spanish Experience}

\author{
Francisco Herrera-Gómez****, F. Javier Álvarez*****. \\ * Farmacología, Facultad de Medicina, Universidad de Valladolid, Valladolid. ** Nefrología, Hospital Virgen \\ de la Concha - Sanidad de Castilla y León, Zamora. *** CEIm, Hospital Clínico Universitario de Valladolid - Sanidad \\ de Castilla y León, Valladolid.
}

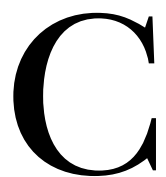
onducir bajo la influencia de drogas ilegales, y algunas substancias legales, no esta permitido a nivel internacional (WHO, 2016). Para evitar esta conducta de riesgo, España es uno de los países de la Unión Europea con "tolerancia cero" (no se permite conducir con ninguna cantidad de droga en el organismo). Las pruebas de control de drogas en carretera (de screening o detección) son obligatorias (la ley sanciona a todos los que se nieguen a someterse a dichas pruebas como si estas pruebas fuesen positivas), y se realizan en fluido oral. Cuando un conductor da positivo en estas pruebas, se obtiene una segunda muestra de fluido oral que se envía a laboratorios acreditados para el análisis de confirmación y cuantificación de las sustancias detectadas (Alvarez, Gonzalez-Luque y Seguí, 2016).

La legislación española establece que cuando la sustancia detectada es un medicamento que precisa de receta médica y se utiliza de acuerdo con su autorización de comercialización (como se indica en el resumen de las características del producto), no se aplican las sanciones establecidas, siempre y cuando no se detecte otra sustancia "no permitida". Esto requiere de un informe (médico) que confirme la prescripción del medicamento detectado (Fierro, Colas, González-Luque y Álvarez, 2017): este podría ser el caso de los paciente en un programa de mantenimiento con metadona.

Recientemente, hemos publicado la presencia de opioides en el análisis de confirmación de aquellos conductores con un resultado positivo en las pruebas de drogas en carretera durante el periodo 2011-2016 (Herrera-Gómez,
García-Mingo, Colas, González-Luque y Alvarez, 2018). En dicho periodo se realizaron un total de 179645 pruebas, 65244 de las cuales fueron positivas. Se confirmó la presencia de metadona en el $4,1 \%$ de todos estos casos positivos, siendo la mayoría conductores varones $(94 \%)$ y de mediana edad (media \pm desviación estándar (DE), $41.90 \pm 7.61$ años). En casi todos los casos, también se detectaron otras drogas ilícitas $(99,6 \%)$, como heroína $(81,9 \%)$, cocaína (45\%), cocaína y cannabis $(33 \%)$, o cannabis sólo $(6 \%)$. En la tabla 1 se muestran las cifras de positivos durante el período cubierto por el estudio.

Tabla 1. Presencia de metadona en los controles de drogas en carretera en España entre 2011-2016

\begin{tabular}{lllll}
\hline & $\begin{array}{l}\text { Número de } \\
\text { pruebas } \\
\text { Año }\end{array}$ & $\begin{array}{l}\text { Número } \\
\text { de casos } \\
\text { los controles } \\
\text { de drogas en } \\
\text { carretera* }\end{array}$ & $\begin{array}{l}\text { los controles } \\
\text { les drogas en } \\
\text { carretera** }\end{array}$ & $\begin{array}{l}\text { Número de casos confirmados } \\
\text { en el laboratorio de toxicología }\end{array}$ \\
\cline { 5 - 5 } & $\begin{array}{l}\text { Metadona } \\
\text { sola }\end{array}$ & $\begin{array}{l}\text { Metadona y } \\
\text { otras sustancias }\end{array}$ \\
\hline 2011 & 743 & 62 & 0 & 10 \\
2012 & 3487 & 1087 & 1 & 169 \\
2013 & 4563 & 2017 & 1 & 132 \\
2014 & 29643 & 9991 & 2 & 465 \\
2015 & 76040 & 25966 & 3 & 933 \\
2016 & 65169 & 26121 & 5 & 935 \\
\hline Total & 179645 & 65244 & 12 & 2644 \\
\hline
\end{tabular}

Nota. ${ }^{*}$ Los datos corresponden al número de pruebas por cada año

** Los datos se refieren a los casos positivos en los controles de screening o detección de drogas en carretera 
Los resultados de este estudio enfatizan el grave problema del uso de múltiples sustancias psicoactivas por los conductores Españoles. La metadona y otros opioides autorizados como medicamentos, con frecuencia son utilizados con fines de abuso y, por lo general, en un uso combinado con otras sustancias psicoactivas por parte de la población (Guardia, 2018; Volkow y McLellan, 2016).

Un aspecto clave constituye el qué hacer cuando un conductor-paciente solicita un informe (médico) que confirme la prescripción de metadona (y otros fármacos) para evitar una sanción de tráfico tras un resultado positivo en un control de drogas. Debería ser la norma el solicitar a estos pacientes el documento remitido por las autoridades de tráfico con el resultado del análisis de confirmación, antes de emitir el informe confirmando la prescripción de metadona (y otros fármacos), si ese es el caso. No debe olvidarse que el conductor-paciente será sancionado si se detectan otras sustancias psicoactivas no legalmente autorizadas en un conductor. En cualquier caso, siempre debemos informar de lo que conocemos: el medicamento recetado y el estado clínico del paciente.

Los kits actualmente utilizados en los controles de drogas en carretera en España no detectan metadona, y, por lo tanto, no debe sorprender que un gran porcentaje de conductores positivos a metadona también fuera positivo a otros opioides (heroína) y drogas ilícitas (cocaína, cannabis) en el análisis de confirmación. Sin embargo, los elevados porcentajes de uso combinado nos sorprendieron y los consideramos alarmantes.

En Europa, de los pacientes en programa de sustitución a opioides, el $63 \%$ se encuentran en tratamiento con metadona, mientras que el $35 \%$ está siendo tratado con buprenorfina y buprenorfina/naloxona (EMCDDA, 2017, pp: 68-69). En España, en 2016, el 91,2\% estaba en tratamiento con metadona, mientras que sólo el $8,8 \%$ con buprenorfina/naloxona (Plan Nacional sobre Drogas, 2018, pp: 109-110). Buprenorfina y metadona pueden ocasionar un deterioro de moderado a grave en la capacidad para conducir con seguridad (Ravera et al, 2013), y, como otros medicamentos que afectan a la conducción, los envases de estos medicamentos en España están provistos de un pictograma específico: el pictograma "medicamentos y conducción” (y en los prospectos también se incluye información para el paciente sobre estos efectos).

Los datos no permiten determinar si estos casos positivos a metadona en controles de drogas en carretera corresponden, o no, a conductores de vehículos que "abusan" de este opioide, o se trata de conductores en programas de mantenimiento con metadona. Hay que tener en cuenta la frecuente comorbilidad de estos pacientes dependientes a opioides, que pudiera afectar también su aptitud para conducir. Por lo tanto, la selección del medicamento(s) que ocasione menos deterioro al conductor es particularmente relevante en estos casos.
Se debe informar a los pacientes que si conducen con la presencia de drogas están adoptando una conducta de riesgo para ellos y para el resto de usuarios de las vías, y que los dispositivos actuales detectan la presencia de drogas (y ciertos medicamentos) en saliva, y que podrían ser sancionados (Alvarez, Gonzalez-Luque y Seguí, 2016 ; Gutiérrez-Abejón, Herrera-Gómez, Criado-Espegel y Álvarez, 2017).

\section{Conflicto de intereses}

El Dr. F. Javier Alvarez ha recibido ayudas y se ha desempeñado como consultor, asesor, u orador de Formación Continuada durante los últimos cuatro años para las siguientes entidades: Reckitt Benckiser, Indivior y Shire.

Financiación

Este trabajo ha sido financiado por el Instituto de Salud Carlos III, Redes Temáticas de Investigación Cooperativa, Red de Trastornos Adictivos [número de concesión RD16/0017/0006], co-financiado por los fondos FEDER de la Unión Europea: una manera de hacer Europa.

\section{Referencias}

Álvarez, F.J., González-Luque, J.C. y Seguí-Gómez, M. (2015). Drugs, Substance Use Disorder and Driving: Intervention of Health Professionals in the Treatment of Addictions. Adicciones 27, 161-167. doi:10.20882/adicciones.702.

EMCDDA. (2017). European Drug Report 2017. Lisbon: EMCDDA. Recuperado de http://www.emcdda.europa. eu/edr2017.

Fierro, I., Colás, M., González-Luque, J.C. y Álvarez, F.J. (2017). Roadside opioid testing of drivers using oral fluid: the case of a country with a zero tolerance law, Spain. Substance Abuse Treatment, Prevention, and Policy, 12, 22. doi:10.1186/s13011-017-0108-3.

Guardia, J. (2018). Overdose epidemic linked to the prescription of opioid analgesics in the United States. Adicciones, 30, 87-92. doi:10.20882/adicciones.936.

Gutierrez-Abejón, E., Herrera-Gómez, F., Criado-Espegel, P. y Álvarez, F.J. (2017). Use of driving-impairing medicines by the population: a population-based registry study. BMJ Open, 7, e017618. doi:10.1136/bmjopen-2017-017618.

Herrera-Gómez, F., García-Mingo, M., Colás, M., González-Luque, J.C. y Álvarez FJ. (2018). Opioids in oral fluid of Spanish drivers. Drug and Alcohol Dependence, 187, 3539. doi:10.1016/j.drugalcdep.2018.02.016.

Plan Nacional sobre Drogas. (2018). Memoria 2016. Delegación del Gobierno para el Plan Nacional sobre Drogas (DGPNSD). Madrid: Ministerio de Sanidad, Servicios Sociales e Igualdad. Recuperado de http://www.pnsd. mscbs.gob.es/profesionales/publicaciones/catalogo/catalogoPNSD/publicaciones/pdf/2018_MEMORIA_2016.pdf. 
Ravera, S., Monteiro, S., de Gier J. J., van der Linden, T., Gómez-Talegón, T. y Álvarez, F. J., the DRUID Project WP4 Partner (2012). A European approach to categorising medicines for fitness to drive: Outcomes of the DRUID project. British Journal of Clinical Pharmacology, 74, 920-931. doi:10.1111/j.1365-2125.2012.04279.x.
Volkow, N.D. y McLellan, A.T. (2016). Opioid abuse in chronic pain-misconceptions and mitigation strategies. The New England Journal of Medicine, 374, 12531263. doi:10.1056/NEJMra150777.

WHO. (2016). Drug use and road safety: a policy brief. Geneva: World Health Organization. Recuperado de http:/ / www.who.int/iris/handle/10665/249533. 